\title{
Effects of oncological treatments on semen quality in patients with testicular neoplasia or lymphoproliferative disorders
}

\begin{abstract}
Cataldo Di Bisceglie, Angela Bertagna, Emanuela R Composto, Fabio Lanfranco, Matteo Baldi, Giovanna Motta, Anna M Barberis, Emanuela Napolitano, Elena Castellano and Chiara Manieri

Pretherapy sperm cryopreservation in young men is currently included in good clinical practice guidelines for cancer patients. The aim of this paper is to outline the effects of different oncological treatments on semen quality in patients with testicular neoplasia or lymphoproliferative disorders, based on an 8-year experience of the Cryopreservation Centre of a large public hospital. Two hundred and sixty-one patients with testicular neoplasia and 219 patients with lymphoproliferative disorders who underwent chemotherapy and/or radiotherapy and pretherapy semen cryopreservation were evaluated. Sperm and hormonal parameters (follicle-stimulating hormone (FSH), luteinizing hormone (LH), testosterone, inhibin B levels) were assessed prior to and 6, 12, 18, 24 and 36 months after the end of cancer treatment. At the time of sperm collection, baseline FSH level and sperm concentration were impaired to a greater extent in patients with malignant testicular neoplasias than in patients with lymphoproliferative disorders. Toxic effects on spermatogenesis were still evident at 6 and 12 months after the end of cancer therapies, while an improvement of seminal parameters was observed after 18 months. In conclusion, an overall increase in sperm concentration was recorded about 18 months after the end of cancer treatments in the majority of patients, even if it was not possible to predict the evolution of each single case 'a priori'. For this reason, pretherapy semen cryopreservation should be considered in all young cancer patients.
\end{abstract}

Asian Journal of Andrology (2013) 15, 425-429; doi:10.1038/aja.2012.171; published online 1 April 2013

Keywords: chemoradiotherapy; lymphoproliferative disorders; semen analysis; semen cryopreservation; testicular neoplasia

\section{INTRODUCTION}

In the last 10 years, following the increased success rate of cancer treatments, great efforts have been made to improve quality of life in survivors, including fertility preservation in young patients. Because of their gonadotoxic effects, chemo- and radiotherapy can temporarily or permanently compromise fertility. The development of several assisted reproduction techniques, including semen cryopreservation, has brought effective and qualitative changes in this field. ${ }^{1,2}$

Every year 6500 men aged 15-39 years develop cancer in Italy. In particular, malignant testicular neoplasias (TN) represent $16 \%$ of all new cancer diagnoses (incidence of 10.2/100 000 inhabitants), followed by Hodgkin lymphomas and non-Hodgkin lymphomas (respective incidence of 5.0/100 000 and 6.2/100 000). Thanks to modern cancer therapies, these neoplasms are usually associated with good prognosis. Chemotherapy with platinum and its derivatives, surgical treatments and radiotherapy lead to an overall 5-year survival rate of $90 \%-95 \%$ for TN, $92 \%$ for Hodgkin lymphoma and $71 \%$ for non-Hodgkin lymphoma. ${ }^{3,4}$

At the same time, the increase in the overall survival rate of patients with germinal and hematological neoplasias treated with chemo- and radiotherapies is accompanied by an increase in infertility rates. ${ }^{5,6}$
Indeed, oncological treatments present severe gonadotoxic effects on both germ and Leydig cells. ${ }^{7-9}$ Of note, in a significant percentage of patients $(20 \%-50 \%)$ spermatogenesis is impaired even before cancer treatments, probably due to the malignancy itself. ${ }^{10}$ The recovery of normal spermatogenesis after treatment may require several years, and mainly depends on three factors: initial sperm count, type and dose of specific oncological treatments and patient age.

These data justify the increasing efforts in identifying prevention and treatment strategies to preserve reproductive functions in young men with malignancies. In several countries, sperm cryopreservation before gonadotoxic therapy is a common practice for the preservation of reproductive potential in cancer treatment survivors, and specific procedures are well established. ${ }^{1-13}$

The aim of this study was to evaluate the effects of chemotherapy and radiotherapy on seminal and reproductive hormones parameters in patients with malignant testicular tumors or lymphoproliferative disorders.

\section{MATERIALS AND METHODS}

Four hundred and eighty patients who referred to the Sperm Cryopreservation Centre of San Giovanni Battista Hospital, Turin, 
Italy, from October 2000 to April 2008 and who completed a follow-up period of 36 months were included in the study. Of these, 261 patients were affected by TN (mean age \pm s.e.m.: $27.9 \pm 0.6$ years) and were evaluated after unilateral orchiectomy (pT1 stage in $51 \%$ of cases, pT2 in 44\% and pT3 in 5\%): 154 patients (59\%) (TNCT group) were diagnosed with non-seminomatous TN and were treated only with chemotherapy (consisting of 2-4 cycles of bleomycin, etoposide, and cisplatin); 107 patients (41\%) (TNRT group) were affected by seminoma and were treated with radiotherapy only (20-30 Gy dose, directed to the paraaortic/paracaval lymph nodes); 219 patients had malignant lymphoproliferative disorders (LDs; mean age \pm s.e.m.: $29.1 \pm 1.2$ years). Of these, 125 (57\%) (LDCT group) presented with Hodgkin lymphoma and were treated only with chemotherapy (consisting of 4-6 cycles of doxorubicin, bleomycin, vinblastine, and dacarbazine). Ninety-four (43\%) (LDRT group) presented with non-Hodgkin lymphoma and were treated with chemotherapy (consisting of 3-6 cycles of cyclophosphamide, doxorubicin, vincristine, and prednisone/prednisolone plus rituximab in B-cell lymphomas) and radiotherapy (30-36 Gy dose directed towards the chest or abdomen). No detrimental effects of therapy on ejaculation were present in all the patients.

We included in the study only patients with similar diseases and comparable treatments (only chemotherapy or radiotherapy; both chemotherapy and radiotherapy) to limit confounding factors. Patients with TN who underwent retroperitoneal lymph node dissection or surveillance were excluded. Similarly, patients with TN or LD who underwent second line treatment for relapse were excluded.

In all patients, semen parameters (including sperm concentration, progressive motility and sperm morphology) along with hormonal levels (including follicle-stimulating hormone (FSH), luteinizing hormone (LH), testosterone and inhibin B) were assessed at the time of semen cryopreservation before cancer treatments and 6, 12, 18, 24 and 36 months after the end of treatment.

FSH and LH levels were evaluated by immunoradiometric assay, testosterone levels by radioimmunoassay and inhibin B levels by enzyme-linked immunosorbent assay. Semen parameters were evaluated following World Health Organization guidelines for semen analysis. $^{14}$

Seminal and hormonal parameters were compared in each group and among groups during follow-up. Results are expressed as mean \pm s.e.m. Because data do not follow a normal distribution, statistical analyses were performed using Mann-Whitney non-parametric test for unrelated samples and Wilcoxon test for related samples. Results were considered statistically significant for $P<0.05$.

\section{RESULTS}

When the four groups of patients were compared, the TNCT group presented the lowest baseline sperm concentration $(P<0.001, \mathrm{MW})$. LDCT and LDRT presented similar baseline levels, that were higher than the other two groups $(P<0.001, \mathrm{MW})$. TNRT group showed the highest baseline FSH levels $(P<0.001$, MW), while FSH levels in the other three groups were similar. Baseline values of inhibin B were higher in LDCT and LDRT groups than TNCT and TNRT groups $(P<0.001, \mathrm{MW}$; Tables 1-4).

Baseline LH and testosterone levels, sperm progressive motility and normal morphology did not show any difference in the four groups (Table 5).

Patients of the TNCT group showed a reduction in sperm concentration $(P<0.05, \mathrm{~W})$ at 6 and 12 months after treatment, followed by a return to baseline levels at 18, 24 and 36 months after treatment (Table 1 and Figure 1). In this group, FSH levels increased after chemotherapy and continued to be persistently elevated until month $24(P<0.05, \mathrm{~W}$; Table 1 and Figure 2$)$. Inhibin B mirrored FSH variations, being reduced until month $24(P<0.05$, W; Table 1 and Figure 3).

Sperm concentration of TNRT patients underwent no changes during the entire 36 months follow-up (Table 2 and Figure 1). FSH raised at month $6(P<0.01, \mathrm{~W})$ and returned to baseline values by month 12 (Table 2 and Figure 2), while a significant reduction of inhibin B was shown only at 12 months $(P<0.001, \mathrm{~W}$; Table 2 and Figure 3$)$.

The LDCT group showed a reduction in sperm concentration at months 6 and $12(P<0.05, \mathrm{~W})$ and a recovery at months 18,24 and 36 (Table 3 and Figure 1). FSH levels were increased at months 6, 12, 18 and $24(P<0.01, \mathrm{~W}$; Table 3 and Figure 2). Inhibin B levels showed a reduction at month $6(P<0.001, \mathrm{~W})$, but no other statistically significant variations (compared to baseline values) at the other time points (Table 3 and Figure 3).

LDRT group presented a reduction of sperm concentration at months 6 and $12(P<0.01, \mathrm{~W})$, followed by the return to baseline values starting at months 18, 24 and 36 (Table 4 and Figure 1). FSH increased from month $6(P<0.05, \mathrm{~W})$ and returned to baseline levels at months 18 and 36 (Table 4 and Figure 2); on the other hand, inhibin $B$ showed a reduction at month $6(P<0.01, \mathrm{~W})$ and a return to baseline levels at month 18 (Table 4 and Figure 3).

The comparison of hormonal and seminal parameters during follow-up period in the four groups, showed the lowest sperm concentration in TNCT group $(P<0.05, \mathrm{MW})$ at months 6 and 12 . Afterwards, no differences in the increase of sperm concentration were recorded among the four groups when compared to baseline values (Figure 1).

As described before, FSH increase in TNCT and LDCT groups was prolonged until month 24, while in TNRT and LDRT groups, FSH levels returned to baseline values at months 12 and 18-36, respectively (Figure 2).

Inhibin B concentrations remained similar in the four groups at months 6, 12, 18 and 24, while at month 36, inhibin B levels were lower in TNCT group than TNRT group $(P<0.05$, MW; Figure 3$)$.

$\mathrm{LH}$ and testosterone levels, sperm progressive motility and normal morphology did not show any variations at each time point in the different groups.

Table 1 Follicle-stimulating hormone (FSH) and inhibin B levels and sperm concentration (mean \pm s.e.m.) in patients with TNCT in baseline conditions and 6,12, 18, 24 and 36 months after the end of the oncological treatments: in parentheses percent of patients with normal values

\begin{tabular}{|c|c|c|c|c|c|c|c|}
\hline & \multirow[b]{2}{*}{ Normal value } & \multicolumn{6}{|c|}{ Month after oncological treatments } \\
\hline & & 0 & 6 & 12 & 18 & 24 & 36 \\
\hline $\mathrm{FSH}\left(\mathrm{mlU} \mathrm{ml}^{-1}\right)$ & $<10$ & $6.5 \pm 0.5(79 \%)$ & $20.4 \pm 2.2(19 \%)$ & $18.5 \pm 1.8(21 \%)$ & $14.5 \pm 2.9(44 \%)$ & $18.3 \pm 3.2(30 \%)$ & $13.5 \pm 1.5(50 \%)$ \\
\hline Inhibin B (pg ml ${ }^{-1}$ ) & $\geqslant 80$ & $56.5 \pm 2.8(22 \%)$ & $36.2 \pm 3.8(3 \%)$ & $38.3 \pm 4.6(10 \%)$ & $58.3 \pm 10.3(24 \%)$ & $37.8 \pm 6.2(11 \%)$ & $52.2 \pm 6.8(21 \%)$ \\
\hline Sperm concentration (million $\mathrm{ml}^{-1}$ ) & $\geqslant 20$ & $33.1 \pm 3.2(62 \%)$ & $19.5 \pm 6.2(32 \%)$ & $15.5 \pm 3.5(28 \%)$ & $48.3 \pm 14.0(62 \%)$ & $42.8 \pm 10.2(60 \%)$ & $42.7 \pm 13.2(61 \%)$ \\
\hline
\end{tabular}


Table 2 Follicle-stimulating hormone (FSH) and inhibin B levels and sperm concentration (mean \pm s.e.m.) in patients with TNRT in baseline conditions and $6,12,18,24$ and 36 months after the end of the oncological treatments: in parentheses percent of patients with normal values

\begin{tabular}{|c|c|c|c|c|c|c|c|}
\hline & \multirow[b]{2}{*}{ Normal value } & \multicolumn{6}{|c|}{ Month after oncological treatments } \\
\hline & & 0 & 6 & 12 & 18 & 24 & 36 \\
\hline 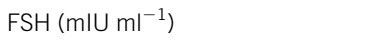 & $<10$ & $9.5 \pm 0.5(55 \%)$ & $15.9 \pm 2.0(34 \%)$ & $11.6 \pm 1.4(54 \%)$ & $11.2 \pm 2.8(50 \%)$ & $8.4 \pm 0.9(67 \%)$ & $8.7 \pm 1.6(70 \%)$ \\
\hline Inhibin B (pg ml $\left.{ }^{-1}\right)$ & $\geqslant 80$ & $58.5 \pm 3.5(24 \%)$ & $49.6 \pm 7.4(16 \%)$ & $44.0 \pm 4.8(11 \%)$ & $59.7 \pm 6.3(25 \%)$ & $55.6 \pm 5.8(23 \%)$ & $71.5 \pm 6.5(40 \%)$ \\
\hline Sperm concentration (million $\mathrm{ml}^{-1}$ ) & $\geqslant 20$ & $46.0 \pm 4.5(66 \%)$ & $36.2 \pm 6.4(47 \%)$ & $43.8 \pm 8.1(63 \%)$ & $56.8 \pm 16.3(80 \%)$ & $54.0 \pm 9.6(82 \%)$ & $52.3 \pm 11.3(84 \%)$ \\
\hline
\end{tabular}

Table 3 Follicle-stimulating hormone (FSH) and inhibin B levels and sperm concentration (mean \pm s.e.m.) in patients with LDCT in baseline conditions and $6,12,18,24$ and 36 months after the end of the oncological treatments: in parentheses percent of patients with normal values

\begin{tabular}{|c|c|c|c|c|c|c|c|}
\hline & \multirow[b]{2}{*}{ Normal value } & \multicolumn{6}{|c|}{ Month after oncological treatments } \\
\hline & & 0 & 6 & 12 & 18 & 24 & 36 \\
\hline $\mathrm{FSH}\left(\mathrm{mlU} \mathrm{ml} \mathrm{l}^{-1}\right)$ & $<10$ & $5.0 \pm 0.3(92 \%)$ & $11.3 \pm 1.5(50 \%)$ & $8.6 \pm 1.7(57 \%)$ & $12.2 \pm 3.9(49 \%)$ & $10.9 \pm 2.1(52 \%)$ & $8.4 \pm 2.1(73 \%)$ \\
\hline Inhibin B (pg ml ${ }^{-1}$ ) & $\geqslant 80$ & $94.8 \pm 4.8(50 \%)$ & $47.2 \pm 5.3(12 \%)$ & $63.2 \pm 9.3(38 \%)$ & $74.1 \pm 16.3(40 \%)$ & $66.3 \pm 10.0(45 \%)$ & $73.3 \pm 12.5(55 \%)$ \\
\hline Sperm concentration (million $\mathrm{ml}^{-1}$ ) & $\geqslant 20$ & $76.3 \pm 6.8(73 \%)$ & $32.8 \pm 8.3(46 \%)$ & $21.9 \pm 5.6(36 \%)$ & $43.9 \pm 13.5(64 \%)$ & $50.3 \pm 15.9(80 \%)$ & $48.1 \pm 8.8(83 \%)$ \\
\hline
\end{tabular}

Table 4 Follicle-stimulating hormone (FSH) and inhibin B levels and sperm concentration (mean \pm s.e.m.) in patients with LDRT in baseline conditions and $6,12,18,24$ and 36 months after the end of the oncological treatments: in parentheses percent of patients with normal values

\begin{tabular}{|c|c|c|c|c|c|c|c|}
\hline & \multirow[b]{2}{*}{ Normal value } & \multicolumn{6}{|c|}{ Month after oncological treatments } \\
\hline & & 0 & 6 & 12 & 18 & 24 & 36 \\
\hline $\mathrm{FSH}\left(\mathrm{mlU} \mathrm{ml^{-1 } )}\right.$ & $<10$ & $4.7 \pm 0.4(96 \%)$ & $9.8 \pm 1.3(58 \%)$ & $12.1 \pm 2.7(50 \%)$ & $4.8 \pm 1.0(80 \%)$ & $11.8 \pm 4.0(55 \%)$ & $5.8 \pm 1.3(83 \%)$ \\
\hline Inhibin B (pg ml $\left.{ }^{-1}\right)$ & $\geqslant 80$ & $107.2 \pm 9.5(59 \%)$ & $63.0 \pm 8.7(33 \%)$ & $52.1 \pm 15.3(20 \%)$ & ) $107.7 \pm 23.6$ (67\%) & $58.2 \pm 22.3(50 \%)$ & $74.3 \pm 15.8(60 \%)$ \\
\hline Sperm concentration (million $\mathrm{ml}^{-1}$ ) & $\geqslant 20$ & $74.9 \pm 12.1(75 \%)$ & $47.3 \pm 12.5(57 \%)$ & $32.6 \pm 22.3(45 \%)$ & $102.7 \pm 30.6(73 \%)$ & $49.7 \pm 35.1(77 \%)$ & $69.0 \pm 24.6(78 \%)$ \\
\hline
\end{tabular}

\section{DISCUSSION}

Malignancies and cancer treatments can temporarily or permanently impair male fertility. Predictive markers for gonadal function after oncological treatments are not currently available. The type and duration of treatment can influence post-treatment recovery, even if very different results have been reported in patients undergoing the same therapeutic schemes. ${ }^{15}$ Because innovative fertility preservation techniques (i.e., semen cryopreservation) exist, this aspect should always be evaluated and discussed with patients before the initiation of a cancer treatment, taking into consideration adequate preventive measures and multidisciplinary collaborations with the entire medical staff.

This study shows that baseline FSH levels and sperm concentration were impaired to a greater extent in TN than LD patients. These data are in agreement with previous studies. ${ }^{16,17}$ The lowest baseline sperm concentration was detected in the TNCT group, while the TNRT group showed higher baseline FSH levels when compared to the TNCT group, likely because of the presence of elevated $\beta$-hCG levels with FSH (and spermatogenesis) inhibition in some TNCT patients with non-seminomatous germ cell tumors. ${ }^{18}$ On the other hand, baseline inhibin B levels were higher in LDCT and LDRT groups than in TNCT and TNRT groups.
In transversal and longitudinal trials, some authors evaluated hormonal fluctuations in patients with germ cell tumors after chemotherapy. ${ }^{19}$ A compensated impairment of the Leydig cell function could be observed up to 60 months following chemotherapy. Meanwhile, $68 \%$ of the patients showed increased FSH levels, indicating that spermatogenesis is more severely impaired than steroidogenesis.

The present follow-up data indicate that the increase of FSH levels in TNCT and LDCT was prolonged until month 24, while in TNRT and LDRT groups, FSH levels returned to baseline values at months 12 and 18-36, respectively. Inhibin B concentrations remained similar in the four groups at months 6, 12, 18 and 24, while inhibin B levels were lower in TNCT group at month 36 than in TNRT group. On the other hand, no difference in testosterone and LH levels was detected after cancer therapy.

Gandini et al. ${ }^{20}$ investigated chemotherapy and radiotherapy shortand long-term effects on spermatogenesis in 166 patients with TN with the aim to identify possible correlations among pre-treatment sperm parameters, tumor histotype, treatment and post-treatment spermatogenesis' modifications. A significant worsening of sperm parameters was observed in 71 patients who underwent chemotherapy, and the most important impairments were detected 3 months after the end of treatment. The reduction was even more pronounced at 6

Table 5 Baseline luteinizing hormone (LH) and testosterone levels, sperm progressive motility and normal morphology (mean \pm s.e.m.) in the four groups of patients

\begin{tabular}{lccc}
\hline & $L H\left(m / U m^{-1}\right)$ & Testosterone $\left(\mathrm{ng} \mathrm{ml^{-1 }}\right)$ & Progressive motility (\%) \\
\hline TNCT & $4.0 \pm 0.3$ & $5.4 \pm 0.2$ & $44.5 \pm 1.7$ \\
TNRT & $3.4 \pm 0.2$ & $4.7 \pm 0.2$ & $48.8 \pm 1.9$ \\
LDCT & $3.6 \pm 0.2$ & $4.4 \pm 0.2$ & $40.4 \pm 1.7$ \\
LDRT & $2.9 \pm 0.3$ & $4.8 \pm 0.2$ & $4.4 \pm 1.0$ \\
\hline
\end{tabular}




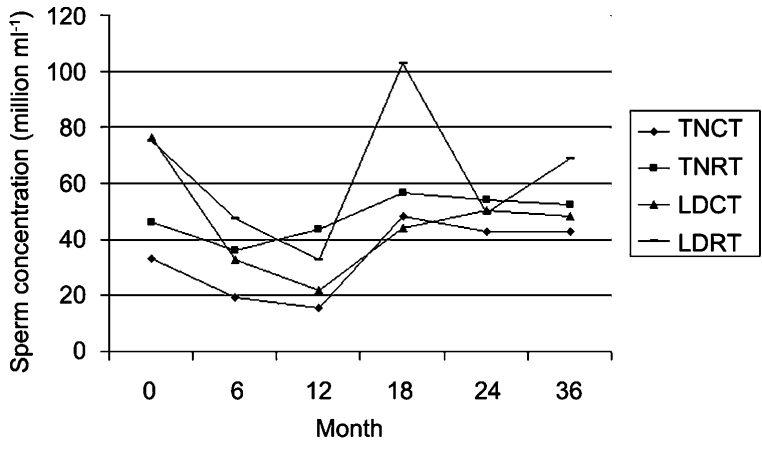

Figure 1 Sperm concentration (million $\mathrm{ml}^{-1}$ ) in baseline conditions and 6, 12, 18,24 and 36 months after the end of the oncological treatments.

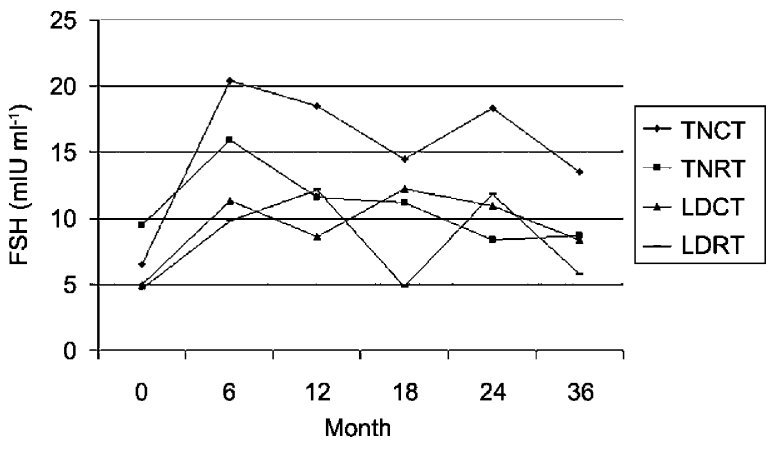

Figure 2 Follicle-stimulating hormone (FSH) level $\left(\mathrm{mIU} \mathrm{ml}^{-1}\right)$ in baseline conditions and 6, 12, 18, 24 and 36 months after the end of the oncological treatments.

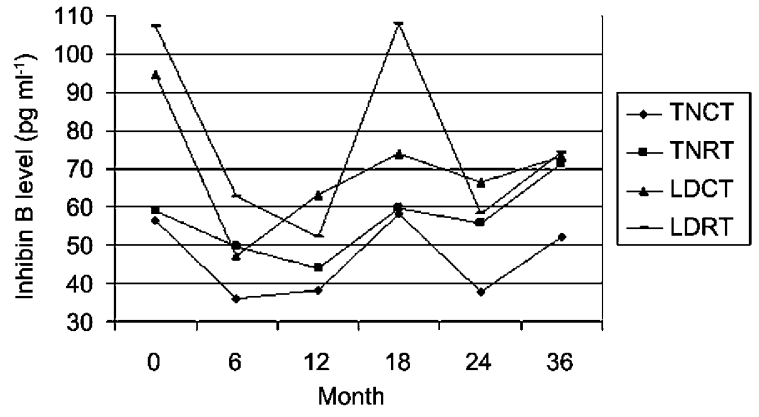

Figure 3 Inhibin B level ( $\mathrm{pg} \mathrm{ml}^{-1}$ ) in baseline conditions and 6, 12, 18, 24 and 36 months after the end of the oncological treatments.

months after the end of treatment in 95 patients who had undergone radiotherapy.

In our study, TNCT, LDCT and LDRT groups showed a significant reduction in sperm concentration at months 6 and 12 after treatment; no significant changes were noticed in TNRT group; sperm motility and morphology showed no differences among the four groups. An improvement of sperm concentration was observed after 18 months in patients who underwent cancer therapy.

Our results confirmed the great impact that chemo- and radiotherapy have on semen quality and sex hormone profile, and, at the same time, the absence of reliable predictors of fertility status after treatment. Recovery of sperm concentration was recorded 18 months after the end of cancer treatments in the majority of the cases, even if it is was not possible to predict the evolution of each single case ' $a$ priori'.

In conclusion, counselling on fertility preservation techniques and andrological follow-up should be offered to all patients in the setting of a specialized cryopreservation centre, in order to monitor semen and hormone modifications over time and to identify possible differences in the evolution of specific malignancies and treatment schemes. Because it is often difficult to have long time follow-ups, it is advisable that cryopreservation centres create an information network in order to allow the comparison of the single centres' experience. This would in turn facilitate future choices regarding the best therapeutic options to be offered to cancer patients, and point out new preventive measures in the field of fertility preservation.

\section{AUTHOR CONTRIBUTIONS}

CDB planned the study, controlled the study cases, performed clinical evaluation of most of the patients, revised all seminal samples and wrote the article with the contribution of CM and FL. ERC, EN and EC helped CDB in clinical evaluation, contacts with the patients and maintenance of data base. MB and GM performed the statistical analyses. $\mathrm{AB}$ and $\mathrm{AMB}$ evaluated all seminal samples according to $\mathrm{WHO}$ manual. FL collaborated with CDB in writing the English version and revising the bibliography. CM collaborated with $\mathrm{CDB}$ in planning, revising and writing the entire paper and is responsible of the collected data. The authors have nothing to disclose about the data presented in this manuscript.

\section{COMPETING FINANCIAL INTERESTS}

We declare that we have no competing financial interests.

\section{ACKNOWLEDGMENTS}

We are grateful to Dr Federica Guaraldi for language editing of the manuscript.

1 Levine J, Canada A, Stern CJ. Fertility preservation in adolescents and young adults with cancer. J Clin Oncol 2003; 28: 4831-41.

2 Ping $\mathrm{P}$, Zhu WB, Zhang XZ, Yao KS, Xu P et al. Sperm banking for male reproductive preservation: a 6-year retrospective multi-centre study in China. Asian J Androl 2010; 12: 356-62.

3 Albers P, Albrecht W, Algaba F, Bokemeyer C, Cohn-Cedermark G et al. Guidelines on testicular cancer. Eur Urol 2005; 48: 885-94.

4 Desandes E. Survival from adolescent cancer. Cancer Treat Rev 2007; 33: 609-15.

5 Trottmann M, Becker AJ, Stadler T, Straub J, Soljanik I et al. Semen quality in men with malignant diseases before and after therapy and the role of cryopreservation. Eur Urol 2007; 52: 355-67.

6 Dohle GR. Male infertility in cancer patients: review of the literature. Int J Uro/2009; 17: 327-31.

7 Colpi GM, Contalbi GF, Nerva F, Sagone P, Piediferro G. Testicular function following chemo-radiotherapy. Eur J Obstet Gynecol Reprod Biol 2004; 113 Suppl 1: S2-6.

8 Howell SJ, Shalet SM. Spermatogenesis after cancer treatment: damage and recovery. J Natl Cancer Inst Monogr 2005; 34: 12-7.

9 Maltaris T, Koelbl H, Seufert R, Kiesewetter F, Beckmann MW et al. Gonadal damage and options for fertility preservation in female and male cancer survivors. Asian $\mathrm{J}$ Androl 2006; 8: 515-33.

10 Petersen PM, Skakkebaek NE, Vistisen K, Rørth M, Giwercman A. Semen quality and reproductive hormones before orchiectomy in men with testicular cancer. J Clin Oncol 1999; 17: 941-7.

11 Lass A, Akagbosu F, Brinsden P. Sperm banking and assisted reproduction treatment for couples following cancer treatment of the male partner. Hum Reprod Update 2001; 7: 370-7.

12 Wallace WH, Anderson RA, Irvine DS. Fertility preservation for young patients with cancer: who is at risk and what can be offered? Lancet Oncol 2005; 6: 209-18.

13 Lee SJ, Schover LR, Partridge AH, Patrizio P, Wallace WH et al. American Society of Clinical Oncology. American Society of Clinical Oncology recommendations on fertility preservation in cancer patients. J Clin Oncol 2006; 24: 2917-31.

14 World Health Organization. WHO Laboratory Manual for the Examination of Human Semen and Sperm-Cervical Mucus Interaction. 4th ed. Cambridge: Cambridge University Press; 1999. 
15 Magelssen H, Haugen TB, von Düring V, Melve KK, Sandstad B et al. Twenty years experience with semen cryopreservation in testicular cancer patients: who needs it? Eur Urol 2005; 48: 779-85.

16 Gandini L, Lombardo F, Salacone P, Paoli D, Anselmo AP et al. Testicular cancer and Hodgkin's disease: evaluation of semen quality. Hum Reprod 2003; 18: 796801.

17 Crha I, Ventruba P, Zakova J, Huser M, Kubesova B et al. Survival and infertility treatment in male cancer patients after sperm banking. Fertil Steril 2009; 91 2344-8.
18 de Bruin D, de Jong IJ, Arts EG, Nuver J, Dullaart RP et al. Semen quality in men with disseminated testicular cancer: relation with human chorionic gonadotropin betasubunit and pituitary gonadal hormones. Fertil Steril 2009; 91: 2481-6.

19 Brennemann W, Stoffel-Wagner B, Helmers A, Mezger J, Jäger N et al. Gonadal function of patients treated with cisplatin based chemotherapy for germ cell cancer. J Urol 1997; 158: 844-50.

20 Gandini L, Sgrò P, Lombardo F, Paoli D, Culasso F et al. Effect of chemo- or radiotherapy on sperm parameters of testicular cancer patients. Hum Reprod 2006; 21: 2882-9. 